\title{
Long-term orbital stability of exosolar planetary systems with highly eccentric orbits
}

\author{
Kyriaki I. Antoniadou ${ }^{1}$ and George Voyatzis ${ }^{2}$
}

Sect. of Astrophysics, Astronomy and Mechanics, Dept. of Physics,

Aristotle University of Thessaloniki, 54124, Greece

${ }^{1}$ email:kyant@auth.gr,

2 email:voyatzis@auth.gr

\begin{abstract}
Nowadays, many extrasolar planetary systems possessing at least one planet on a highly eccentric orbit have been discovered. In this work, we study the possible long-term stability of such systems. We consider the general three body problem as our model. Highly eccentric orbits are out of the Hill stability regions. However, mean motion resonances can provide phase protection and orbits with long-term stability exist. We construct maps of dynamical stability based on the computation of chaotic indicators and we figure out regions in phase space, where the long-term stability is guaranteed. We focus on regions where at least one planet is highly eccentric and attempt to associate them with the existence of stable periodic orbits. The values of the orbital elements, which are derived from observational data, are often given with very large deviations. Generally, phase space regions of high eccentricities are narrow and thus, our dynamical analysis may restrict considerably the valid domain of the system's location.
\end{abstract}

Keywords. dynamical stability, periodic orbits, planetary systems.

With a vast amount of multiple extrasolar systems being discovered over the last two decades, we intend to address the following question: How can highly eccentric resonant exoplanets survive? A dynamical mechanism is offered by mean motion resonances (MMR), which can provide phase protection. Our study is based on this mechanism and aims to show its efficiency. Our methodology breaks down in two steps:

(a) We compute families of periodic orbits in the planar general three body problem, which indicate the exact position of a MMR (e.g. Hadjidemetriou(2006), Antoniadou \& Voyatzis(2013)). We classify them in different configurations (e.g. Antoniadou \& Voyatzis(2014)) and compute their linear horizontal and vertical stability. The vertical critical orbits $(v c o)$ represent the bifurcation points that generate spatial periodic orbits (see Fig. 1a). We justify the MMR and symmetric or asymmetric configuration via librations of resonant angles and apsidal difference about particular angles (Fig. 1b) and depict the planetary evolution (Fig. 1c).

(b) We construct maps of dynamical stability by creating grids defined by two variables (keep the rest orbital elements fixed) and compute the de-trended FLI (Voyatzis (2008)) for $t_{\max }=250 \mathrm{Ky}$ and finally, we visualize its value. The de-trended FLI takes either small values (visualized by dark colors) suggesting regular motion, or very large values (visualized by yellow color) indicating chaotic motion. The distinction between these cases is very sharp when the integration time of orbits is sufficiently large. Certainly, regular orbits guarantee long-term stability of planetary orbits.

In the following figures, we present results for the $2 / 1$ resonant dynamics of the twoplanet system HD 82943b,c (see also Tan et al.(2013), Baluev \& Beaugé(2014)). Maps are computed for the symmetric configuration, which is defined by the resonant angles $\theta_{i}=2 \lambda_{2}-\lambda_{1}-\varpi_{i}=0, i=1,2$, where $\lambda_{i}$ are the mean longitudes and $\varpi_{i}$ the longitudes 
Table 1. Data for the planets HD 82943b,c from Tan et al.(2013).

\begin{tabular}{c|cccccc}
\hline Planets & Mass $\left(M_{\text {Jup }}\right)$ & a $(\mathrm{AU})$ & $\mathrm{e}$ & $\mathrm{i}(\mathrm{deg})$ & $\varpi(\mathrm{deg})$ & $\mathrm{M}(\mathrm{deg})$ \\
\hline HD 82943 c & 4.78 & 1.000 & 0.425 & 19.4 & 133 & 256 \\
HD 82943 b & 4.8 & 1.5951 & 0.203 & 19.4 & 107 & 333 \\
\hline
\end{tabular}
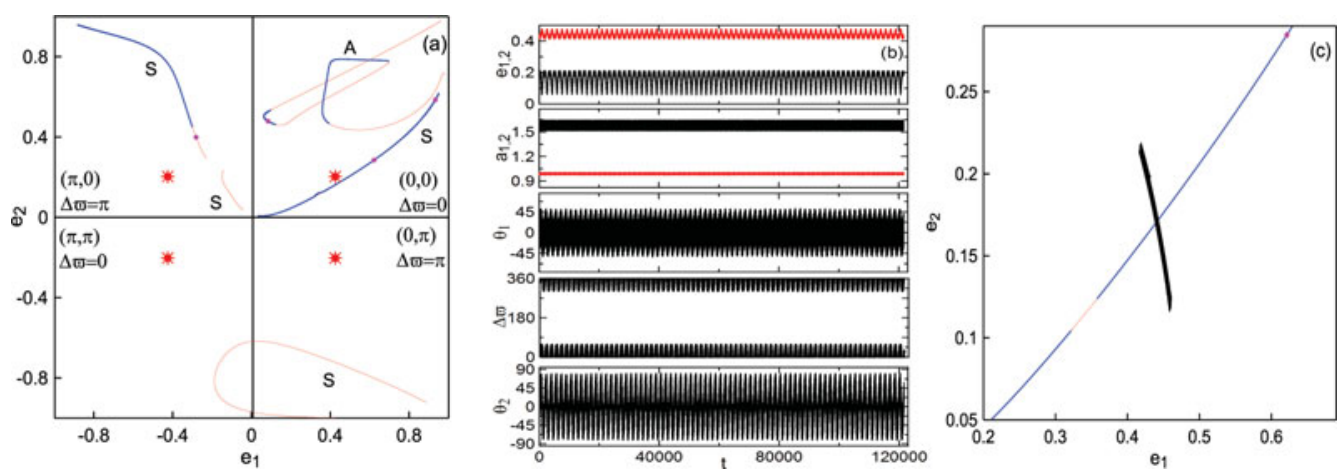

Figure 1. (a) Families of symmetric (S) and asymmetric (A) periodic orbits in $2 / 1 \mathrm{MMR}$ for the planetary mass ratio of HD 82943. Blue (red) segments indicate stable (unstable) orbits. Red stars depict $e_{b, c}$, while magenta dots the vco. (b) Evolution of orbital elements and resonant angles. (c) Projection of the trajectory on the eccentricities plane and the family of periodic orbits.
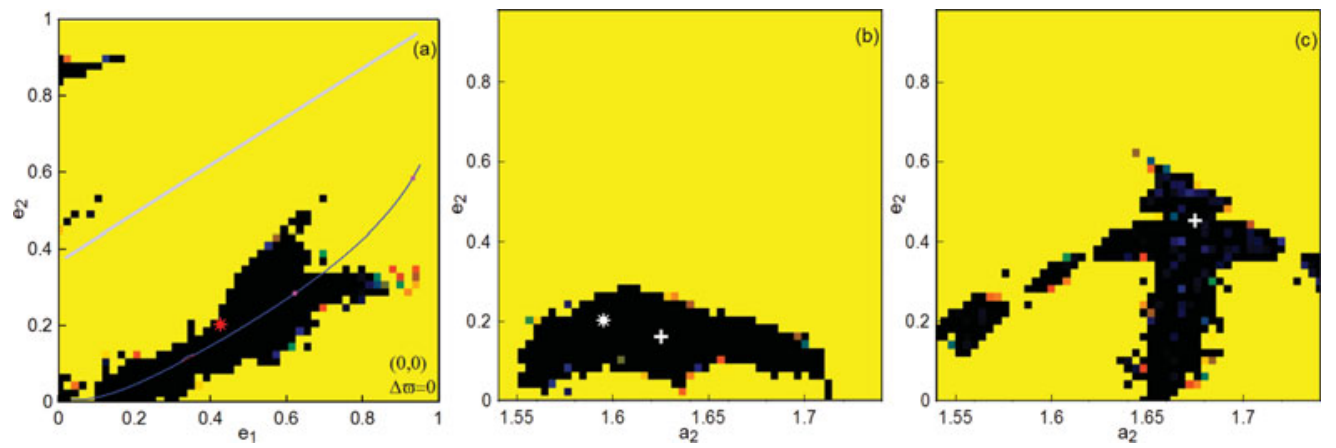

Figure 2. Maps of dynamical stability on: (a) grid plane $\left(e_{1}, e_{2}\right)$ for $a_{2}=1.595$, where the family of periodic orbits and the collision line (bold gray curve) are also presented, (b) $\left(a_{2}, e_{2}\right)$ grid plane for $e_{1}=0.425$, where the resonant periodic orbit is indicated by the cross symbol and the location of HD 82943b,c by a star and (c) as previously, but for $e_{1}=0.8$. In all cases we use the normalized value $a_{1}=1$.

of pericenter. Our analysis shows that the system is located in a region of dynamical stability, which is expanded around a stable periodic orbit. This periodic orbit is vertically stable, thus we may conclude that introducing small mutual inclination the planetary orbits will remain stable.

We have applied the above methodology to the extrasolar systems HD 3651, HD 7449, HD 89744 and HD 102272 and a complete paper is in preparation.

\section{References}

Antoniadou, K. I. \& Voyatzis, G. 2014, Astrophys. Space Sci., 349, 657

Antoniadou, K. I. \& Voyatzis, G. 2013, Celest. Mech. Dyn. Astr., 115, 161

Baluev, R. V. \& Beaugé, C. 2014, MNRAS, 439, 673

Hadjidemetriou, J. D. 2006, Celest. Mech. Dyn. Astr., 95, 225

Tan, X., Payne, M. J., Lee, M. H., et al. 2013, ApJ, 777, 101

Voyatzis, G. 2008, ApJ, 675, 802 